Fukushima J. Med. Sci.,

Vol. 52, No. 2, 2006

[Review Article]

\title{
THE ROLE OF FB21 AS A MARKER OF GLOMERULAR ENDOTHELIAL CELL INJURY
}

\author{
YUKIHIKO KAWASAKI \\ Department of Pediatrics, Fukushima Medical University School of Medicine, \\ Fukushima, 960-1295, Japan
}

(Received June 6, 2006, accepted August 11, 2006)

\begin{abstract}
FB21 was reactive with the glomerular endothelial cells and distal tubules of the human kidney and was bound to a sialic-acid-dependent cell surface antigen. We evaluated the FB21 staining in fetal kidneys, and the kidneys of children and adults with normal kidneys and glomerulonephritis and investigated whether FB21 can be used as a marker for endothelial cell injury. FB21 was reactive with the endothelial cells of normal kidneys and was detected on the surface of endothelial cells by immunoelectron microscopy. FB21 was reactive with endothelial cells in the kidneys of over 32 -week fetuses. The endothelial cell FB21 staining scores in the first renal biopsy specimens of patients with hemolytic uremic syndrome (HUS) were lower than in the kidneys of children with normal kidneys and was negatively correlated with their serum E-selectin concentrations. The FB21 staining of glomerular endothelial cells was similar to the staining for the other endothelial markers, CD34 and von Willebrand factor (vWF). However, FB21 staining of interstitial blood vessels was very weak and was distinct from that of other endothelial markers. These results suggest that FB21 can be used as a specific marker for glomerular endothelial cell injury in various types of glomerulonephritis.
\end{abstract}

Key words: Glomerular endothelium, markers of endothelial cell, FB21, Glomerulonephritis, E-selectin

\section{INTRODUCTION}

Glomerular endothelial cells consist of cell bodies, which are generally located

\footnotetext{
川崎幸彦

Correspondence to: Yukihiko Kawasaki, Department of Pediatrics, Fukushima Medical University School of Medicine, Fukushima City 960-1295, Japan.

E-mail : kyuki@fmu.ac.jp

Dr. Yukihiko Kawasaki received the 2005 Fukushima Society of Medical Science Young Researcher Award.
} 
close to the mesangial axis, and peripherally located attenuated and highly fenestrated cytoplasmic sheets ${ }^{1-3)}$. Glomerular endothelial cells are active participants in the processes controlling coagulation, inflammation, and immune processes, and synthesize and release endothelin-1 and endothelium-derived relaxing factor ${ }^{1,2)}$. Thus, it is suggested that glomerular endothelial cells play an important role in maintaining renal homeostasis.

There had been some reports on endothelial cell injury in various types of glomerulonephritis, such as crescentic glomerulonephritis, hemolytic uremic syndrome (HUS), and lupus nephritis ${ }^{3-6)}$. The tight junctions between endothelial cells and the extracellular matrix tend to be destroyed in pathologic conditions, and desquamation of endothelial cells from the basement membrane tends to occur at the onset of glomerular injury. Endothelial cell injury is important in the pathogenesis and progression of glomerulonephritis.

As indices of glomerular endothelial cell injury, several markers, such as von Willebrand factor (vWF), CD34, and E-Selectin have been used ${ }^{7-12}$. 1. CD34 is a $110 \mathrm{kD}$ transmembrane glycoprotein present on leukemic cells, endothelial cells, and stem cells ${ }^{13-15)}$. Naruse et al. reported that CD34 is a useful marker not of glomerular endothelial cell but of mesangial transformation ${ }^{14,15)}$. VWF is a glycoprotein that mediates platelet adhesion to the subendothelium at sites of vascular injury, and binds and stabilizes factor VIII in the circulation ${ }^{6,13)}$. It appears to be expressed exclusively on endothelial cells, where it exhibits a granular pattern of reactivity. Expression of it is also found in the cytoplasm of megakaryocytes. It is stored in Weibel-Palade bodies. Although it is commonly used as an immunohistochemical marker for endothelial cells, little is known concernings the relative levels of expression of vWF in endothelial cells of the same or different vascular beds in vivo. E-selectin mediates the adhesion of polymorphonuclear cells, monocytes, and some memory $\mathrm{T}$ cells to cytokine-activated endothelial cells ${ }^{5,8}$. Expressed by venular and capillary endothelial cells at sites of active inflammation, it is a marker of endothelial activation in the kidney ${ }^{12)}$.

However, they are not specific markers of only glomerular endothelial cells.

On the other hand, FB21 is a monoclonal antibody that binds a sialic-aciddependent surface antigen and is reactive with human B-cell antigen, glomerular endothelial cells and the epithelial cells of the distal tubules of the human kidney on formalin-fixed, paraffin-embedded tissue sections ${ }^{16,17)}$. We therefore evaluated the FB21 reactivity in fetal kidney and in the kidneys of children and adults with normal kidneys and glomerulonephritis (GN) and investigated whether FB21 can be used as a marker for glomerular endothelial cell injury.

\section{ABOUT FB21}

FB21 was detected by Nozawa et al. as a monoclonal antibody that binds a sialic-acid-dependent surface antigen ${ }^{10,11}$. Nozawa et al. have examined the im- 
munoreactivity of FB21 in a wide range of normal and neoplastic lymphoid tissues, and reported that FB21 is strongly reactive with a subpopulation of B cells in the inner layer of the mantle zone of lymphnodes and weakly reactive with germinal center cells ${ }^{10,11)}$. They also found that FB21 reacts with epithelial cells of the prostate, endometrium, distal renal tubules, and hepatocytes. Immunoprecipitation studies revealed that FB21 may be bound to a sialic-acid-dependent carbohydrate epitope. Sialic acids are derived from neuraminic acid, whose main derivative is $\mathrm{N}$ acetylneuraminic acid, which is generally used as a synonym for sialic acids ${ }^{18)}$. Sialic acids are widely distributed in nature in the form of non-reducing termini of glycoproteins and glycolipids. About $70 \%$ of all sialic acids in eukaryotic cells are found on the cell surface, while the remainder are distributed among the endoplasmic reticulum, mitochondria, and lysosomes. Because of their acidic nature, they impart a negative charge to the cell surface and are important in cell-to-cell and cell-to-matrix interactions.

\section{THE ROLE OF FB21 AS A MARKER OF GLOMERULAR ENDOTHELIAL CELLS}

We found that FB21 was reactive with the glomerular endothelial cells of normal kidney, and was detectable on the surface of endothelial cells on immunogold electron microscopy. However, FB21 was not reactive with glomerular endothelial cells in renal sections after sialidase digestion (Figure 1). These results suggest that glomerular endothelial cells may possess a specific sialic-dependent carbo-

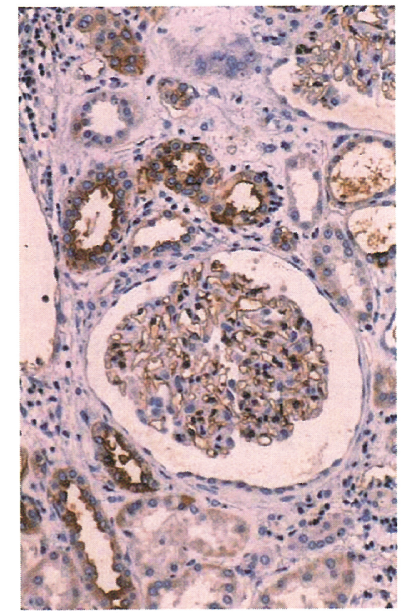

A

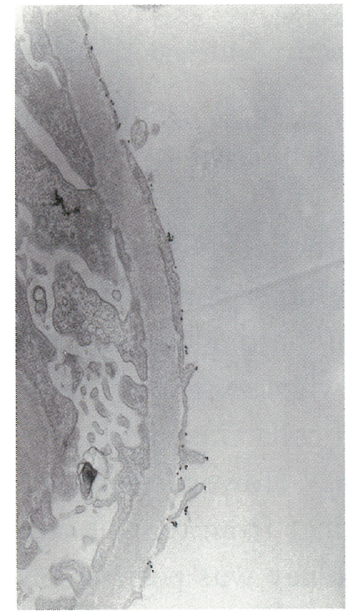

B

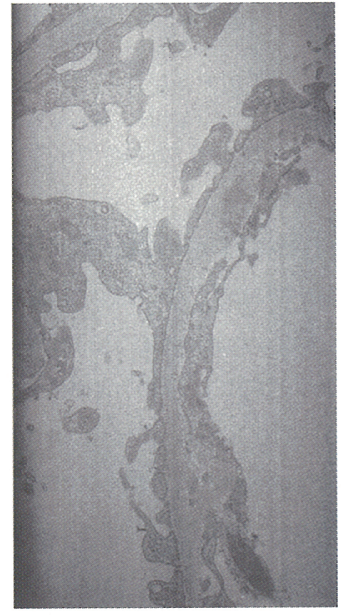

C

Figure 1. Degree of FB21 staining of glomerular endothelial cells in normal kidneys A $(\times 400):$ FB21 is reactive with glomerular endothelial cells on light microscopy.

B $(\times 2500)$. FB21 is detected on the surface of endothelial cells by IEM.

C $(\times 2500)$. FB21 does not react with glomerular endothelial cells in renal sections after sialidase digestion. 


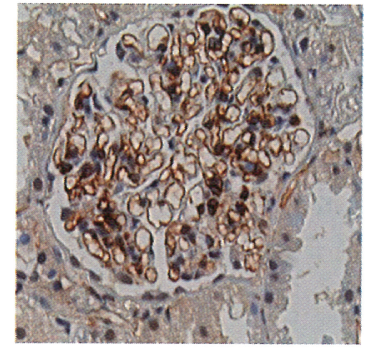

A

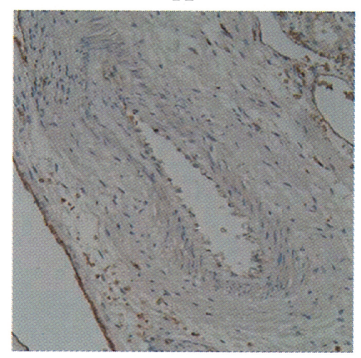

D

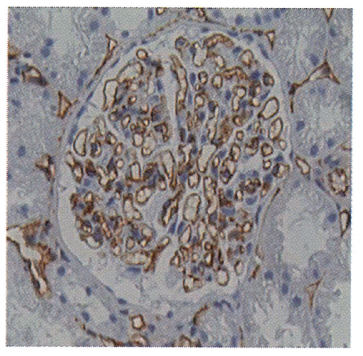

B

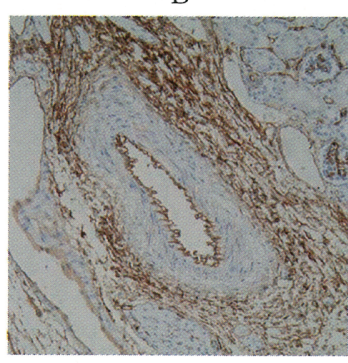

$\mathrm{E}$

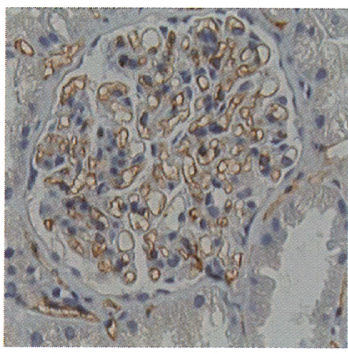

C

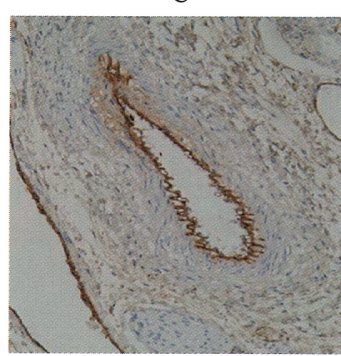

F

Figure 2. Comparison of staining of glomerular endothelial cells for each marker A $(\times 400)$. FB21 is reactive with glomerular endothelial cells on light microscopy.

$\mathrm{B}(\times 400)$. CD34 is reactive with glomerular endothelial cells on light microscopy.

C $(\times 400)$. von Willebrand factor is reactive with glomerular endothelial cells on light microscopy.

D $(\times 400)$. FB21 is not reactive with endothelial cells of interstitial blood vessels on light microscopy.

$\mathrm{E}(\times 400)$. CD34 is reactive with endothelial cells of interstitial blood vessels on light microscopy.

F $(\times 400)$. von Willebrand factor is reactive with endothelial cells of interstitial blood vessels on light microscopy.

hydrate epitope that the endothelial cells of other blood vessels lack ${ }^{12)}$. We compared the results of staining of glomerular endothelial cells with each marker (Figure 2). The endothelial cells of both glomeruli and interstitial blood vessels were reactive for CD34 and vWF when examined by light microscopy. The FB21 staining of glomerular endothelial cells was similar to the staining for the other endothelial markers, CD34 and vWF. However, FB21 staining of interstitial blood vessels was very weak and was distinct from that of other endothelial markers. Staining for FB21 on vessels of the kidney was positive only in glomerular endothelial cells.

In addition, we evaluated FB21 staining in fetal kidneys as well as the kidneys of children with glomerulonephritis including HUS, and investigated whether FB21 can be used as a marker for endothelial cell injury ${ }^{12)}$ (Figures 3, 4, 5).

Based on the characteristic light-microscopic appearance of glomeruli, Naruse identified 4 development stages in the human kidney the vesicle stage, S-shaped body stage, capillary loop stage, and maturing glomerulus stage and showed that fetal 


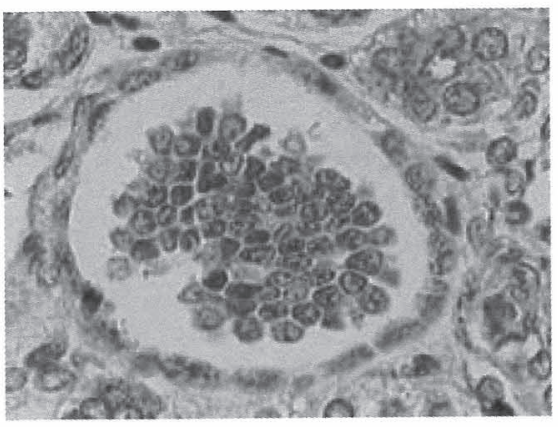

A

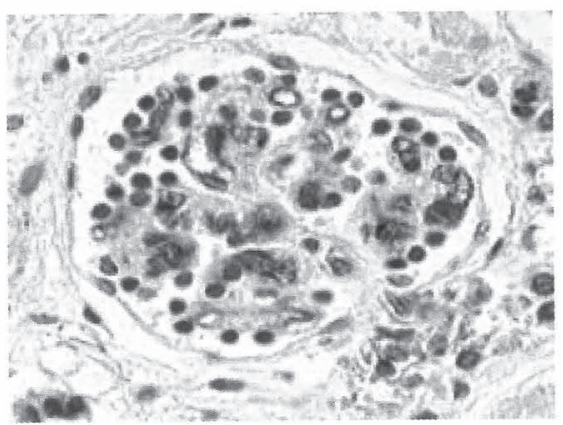

C

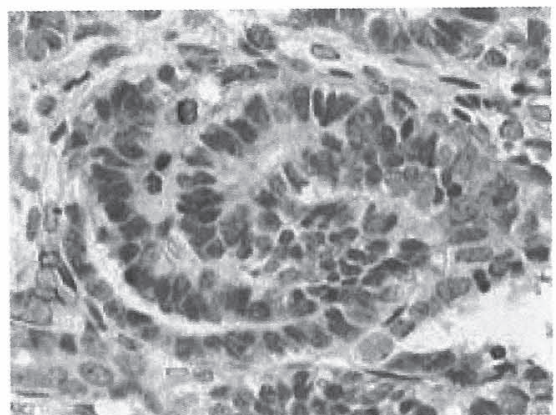

B

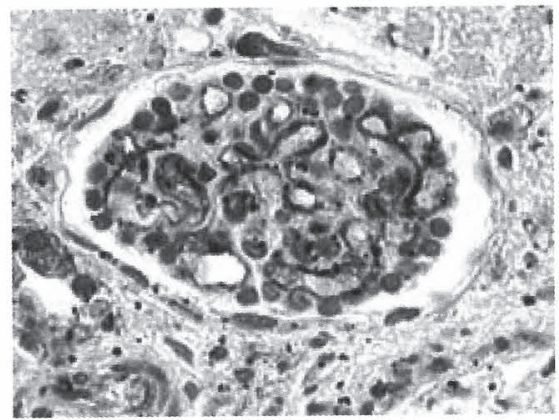

$\mathrm{D}$

Figure 3. FB21 staining of developing fetal kidneys

A $(\times 400)$. There is no FB21 staining in this 20 -week fetal kidney and a mesenchymal cell cluster has formed in the nephrogenic zone.

B $(\times 400)$. There is no FB21 staining in this 26 -week fetal kidney, and the differentiated lower limb of the S-shaped body has formed an immature glomerulus.

C $(\times 400)$. FB21 is weakly reactive with glomerular endothelial cells in this $32-$ week fetal kidney.

D $(\times 400)$. FB21-positive glomerular endothelial cells aggregate at the root of the developing glomerulus and then migrate to the periphery of the glomerular tuft.

glomerular endothelial cells gradually change into adult glomerular endothelial cells in the maturing glomerulus stage ${ }^{14,15}$. We found no reactivity with FB21 in the fetal human kidney before 26 weeks, though FB21 was reactive with glomerular endothelial cells in the fetal kidney after 32 weeks. FB21-positive glomerular endothelial cells later aggregated at the root of the developing glomerulus, and had migrated to the periphery of the glomerular tuft in the 40 -week fetal kidney.

HUS is defined as a triad of microangiopathic hemolytic anemia thrombocytopenia, and acute renal failure. HUS is typical of renal diseases with endothelial cell dysfunction, and microscopic study of renal tissue in HUS shows endothelial swelling and detachment from the basement membrane. We found that FB21 staining of the endothelial cells was significantly weaker in patients with HUS than in children with normal kidneys, and that FB21 staining of glomerular endothelial 


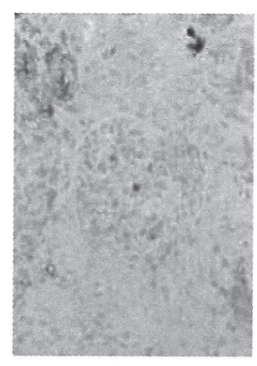

A

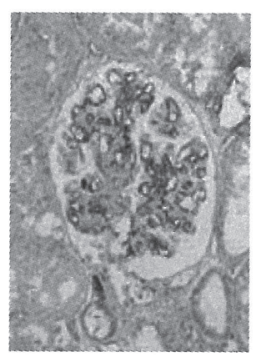

B
Serum E-selectin concentration (pg/ml)

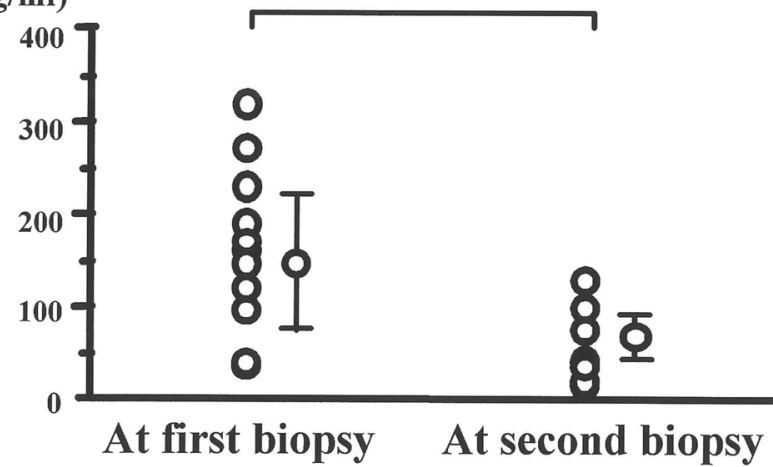

a : $\mathbf{p}<\mathbf{0 . 0 1}$

N.S. not significant

Figure 4. FB21 reactivity with glomerular endothelial cells and serum E-selectin concentrations at first and second biopsies in patients with HSPN. (A) Hardly any FB21 was observed at the time of first biopsy, but (B) FB21 was reactive with glomerular endothelial cells at second biopsy. (C) Comparison of serum Eselectin concentrations at first and second biopsies in patients with HSPN.

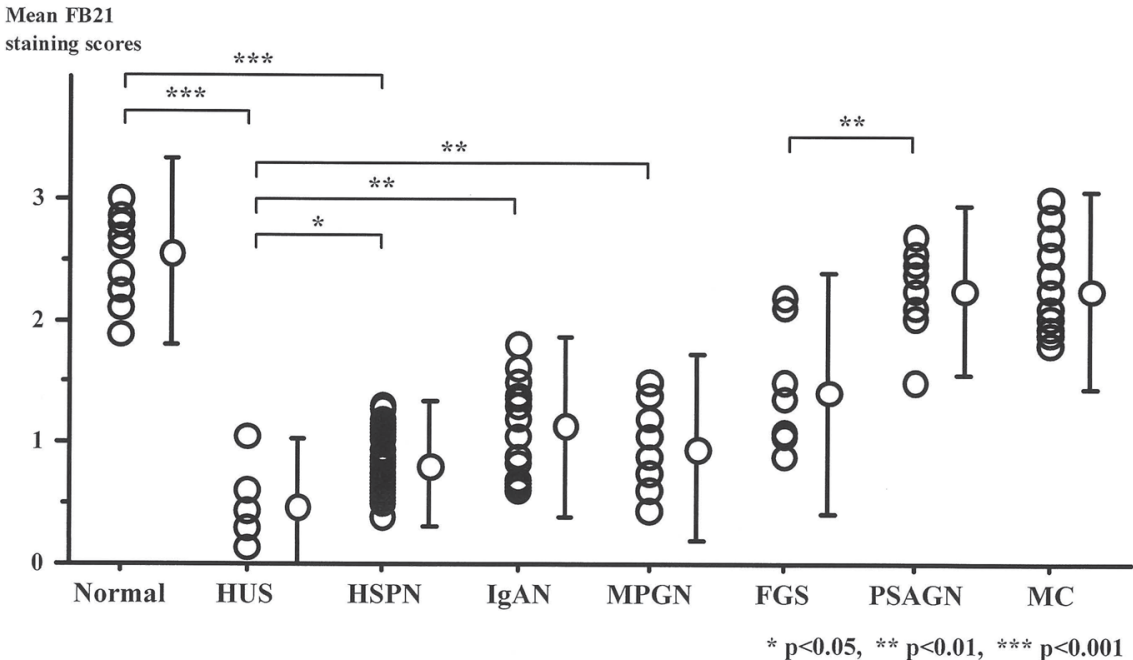

Figure 5. Comparison between mean FB21 staining score of glomelular endothelial cells by form of glomerulonephritis

PSAGN. poststreptococcal acute glomerulonephritis

MC. minimal change 
cells in second biopsy specimens was stronger than that in first biopsy specimens. In addition, serum E-selectin concentrations at the time of first biopsy were greater than at second biopsy, and renal FB21 staining in patients with HUS correlated negatively with serum E-selectin values, an indicator of endothelial cell damage (Figure 4). We therefore concluded that FB21 can be used as a marker for glomerular endothelial cell injury and dysfunction in patients with HUS. In addition, the endothelial cell FB21 staining of crescentic and sclerotic glomerular lesions in patients with immunoglobulin A nephropathy (IgAN), membranoproliferative glomerulonephritis (MPGN), and FGS was weaker than in normal kidneys (Figure 5). We therefore believe that FB21 can be used as a specific marker for glomerular endothelial cell injury in various types of glomerulonephritis ${ }^{12}$.

Mean FB21 staining scores in glomerular endothelial cells in patients with PSAGN and MC were similar to those in normal kidney. PSAGN is characterized by endocapillary proliferative glomerulonephritis and swelling of glomerular endothelial cells. However, the degree of FB21 reactivity with glomerular endothelial cells in PSAGN patients in our study was similar to that in normal kidneys, suggesting that endothelial cell function is retained in PSAGN despite endothelial cell swelling.

The pathogenesis of $\mathrm{MC}$ nephrotic syndrome (MCNS) is related to glomerular epithelial cell injury and the negative charge of glomerular capillaries. We speculated that FB21 staining score of glomerular endothelial cells was lower than in normal kidney because of the relationship between sialic acids and the negative charge of glomerular capillaries. However, our findings in patients with MC with nephrotic syndrome and/or hematuria showed that endothelial cell injury was infrequent, suggesting that the pathogenesis of $\mathrm{MC}$ with nephrotic syndrome and/or hematuria is unrelated to glomerular endothelial cell injury and dysfuction. The sialic-dependent carbohydrate epitope bound to FB21 may thus be distinct from the sialic-dependent carbohydrate epitopes related to the negative charge in patients with $\mathrm{MC}$ and nephrotic syndrome.

Finally, all patients and their parents gave their informed consent in this work and this work was approved by the hospital ethics committee.

\section{THE FUTURE OF FB21}

Although FB21 is used as a specific marker for human glomerular endothelial cell injury and dysfunction, FB21 is not reactive with glomerular endothelial cell of mice, rats, and pigs. Thus, FB21 is not able to use as a glomerular endothelial cell marker in order to investigate the role of glomerular endothelial cell in experimental model of mice, rats, and pigs. In the future, FB21 may be used as an index of distal renal tubules dysfunction, because FB21 reacts with distal renal tubules. 


\section{CONCLUSION}

The reactivity for FB21 of glomerular endothelial cells was similar to those for other endothelial markers, including CD34 and vWF. However, the FB21 staining of interstitial blood vessels was very weak, and distinct from that of other endothelial markers. Staining for FB21 on renal vessels was positive only in glomerular endothelial cells.

We conclude that FB21 can be used as a specific marker for glomerular endothelial cell injury and dysfunction in various forms of glomerulonephritis.

\section{REFERENCES}

1. Savage COS. The biology of the glomerulus: endothelial cells. Kidney Int, 45: $314-$ 319, 1994.

2. Elger M, Sakai T, Kriz W. The vascular pole of the renal glomerulus of rat. Adv Anat Embryol Cel, 139: 1-98, 1998.

3. Savage CO, Pottinger BE, Gaskin G, Pusey CD, Pearson JD. Autoantibodies developing to myeloperoxidase and proteinase 3 in systemic vasculitis stimulate neutrophil cytotoxicity toward cultured endothelial cells. Am J Pathol, 141: 335-342, 1992.

4. Wang MX, Walker RG, Smith PK. Clinicopathologic Associations of anti-endothelial cell antibodies in immunoglobulin A nephropathy and lupus nephritis. Am J Kidney Dis, 22 : 378-386, 1993.

5. Fujieda M, Oishi N, Naruse K, Hashizume M, Nishiya K, Kurashige T, Ito K. Soluble thrombomodulin and antibodies to bovine glomerular endothelial cells in patients with Henoch-Schoenlein purpura. Arch Dis Child, 78 : 240-244, 1998.

6. Remuzzi G, Ruggenenti P. The hemolytic uremic syndrome. Kidney Int, 47 : 2-19, 1995.

7. Wada H, Minamikawa K, Wakita Y, Nakase T, Kaneko T, Ohiwa M, Tamaki S, Deguchi K, Shirakawa S, Hayashi T. Increased vascular endothelial cell markers in patients with disseminated intravascular coagulation. Am J Hematol, 44: 85-88, 1993.

8. Pigott R, Dillon LP, Hemingway IH, Gearing AJH. Soluble forms of E-selectin, ICAM1 and VCAM-1 are present in the supernatants of cytokine-activated endothelial cells. Biochem Bioph Res Co, 187 : 584-589, 1992.

9. Bulger RE, Eknoyan G, Purcell DJ, II, Dobyan DC. Endothelial characteristics of glomeruar capillaries in normal, mercuric chloride-induced, and gentamicin-induced acute renal failure in the rat. J Clin Invest, 72 : 128-141, 1983.

10. Nozawa Y, Abe M, Ohno H, Fukuhara S, Wakasa H. Production of two monoclonal antibodies (FB1 and FB21) useful for the identification of human B lymphocytes in formalin-fixed, paraffin-embedded tissues. J Pathol, 173: 347-354, 1994.

11. Nozawa Y, Wachi E, Tominaga K, Abe M, Wakasa H. A novel monoclonal antibody (FUN-1) identified an activation antigen in cells of the B-cell lineage and Reed-Sternberg cells. J Pathol, 169: 309-315, 1993.

12. Kawasaki Y, Suzuki J, Nozawa R, Sakai N, Tannji M, Isome M, Suzuki H, Nozawa Y. FB21, a monoclonal antibody that reacts with a sialic-acid-dependent carbohydrate epitope, is a marker for glomerular endothelial cell injury. Am J Kidney Dis, 44 : 239 249, 2004.

13. Pusztaszeri M, Chaubert P, Bosman FT. Immunohistochemical expression of endothelial markers CD31, CD34, von Willebrand factor, and fli-1 in normal human tissues. J Histochem Cytochem, 54: 385-395, 2006. 
14. Naruse K, Fujieda M, Miyazaki E, Hayashi Y, Kuroda N, Nakayama H, Kiyoku H, Hiroi M, Kurashige T, Enzan H. DE34 expression as a novel marker of transformed mesangial cells in biopsied glomerular disease. J Pathol, 189: 105-111, 1999.

15. Naruse K, Fujieda M, Miyazaki E, Hayashi Y, Kuroda N, Nakayama H, Kiyoku H, Hiroi M, Kurashige T, Enzan H. An immunohistochemical study of developing glomeruli in human fetal kidneys. Kidney Int, 57: 1836-1846, 2000.

16. Khan BV, Harrison DG, Olbrych MT, Alexander RW, Medford RM. Nitric oxide regulates vascular cell adhesion molecule 1 gene expression and redox-sensitive transcriptional events in human vascular endothelial cells. Proc Natl Acad Sci USA, 93 : 9114-9119, 1996.

17. Janssen-Heininger YM, Poynter ME, Baeuerle PA. Recent advances toward understanding redox mechanisms in the activation of nuclear factor $\varkappa$ B. Free Radical Bio Med, 28 : 1317-1327, 2000.

18. Ledeen RW, Yu RK. Chemistry and analysis of sialic acid. In : Rosenberg A, Schengrund C, eds. Biological Roles of Sialic Acid. Plenum Press, New York, NY, USA., 157, 1976. 